Relations industrielles

Industrial Relations

\title{
Les jeunes et les métiers du bâtiment
}

\section{Marcel Clément}

Volume 4, numéro 6, février 1949

URI : https://id.erudit.org/iderudit/1023476ar

DOI : https://doi.org/10.7202/1023476ar

Aller au sommaire du numéro

Éditeur(s)

Département des relations industrielles de l'Université Laval

ISSN

0034-379X (imprimé)

1703-8138 (numérique)

Découvrir la revue

Citer cet article

Clément, M. (1949). Les jeunes et les métiers du bâtiment. Relations industrielles / Industrial Relations, 4(6), 54-56. https://doi.org/10.7202/1023476ar

Tous droits réservés @ Département des relations industrielles de l’Université Laval, 1949
Ce document est protégé par la loi sur le droit d'auteur. L’utilisation des services d'Érudit (y compris la reproduction) est assujettie à sa politique d'utilisation que vous pouvez consulter en ligne.

https://apropos.erudit.org/fr/usagers/politique-dutilisation/ 


\title{
LES JEUNES ET LES MÉTIERS DU BÂtIMENT
}

\author{
Marcel Clément
}

\section{A-Etablissements des faits}

Au cours de l'enquête entreprise, depuis le mois de juin dernier, sur « la main d'oeuvre dans l'industrie du bâtiment», nous avons été amené à étudier, à la lumière des statistiques, d'une façon toute spéciale, le problème de l'attitude des jeunes devant l'apprentissage dans les métiers de la construction. Avant de tenter toute espèce de commentaire et d'interprétation, et dans le dessein de donner aux faits eux-mêmes leur pleine va leur scientifique, nous commencerons par indiquer les chiffres obtenus.

\section{TABLEAU I}

Classification, par âge et par comité paritaire, des journaliers du bâtiment, au 31 juillet 1948 dans 5 comités paritaires

\begin{tabular}{|c|c|c|c|c|c|c|c|c|}
\hline $\begin{array}{c}\text { Comité } \\
\text { paritaire }\end{array}$ & $\begin{array}{l}\text { moins } \\
\text { de } \\
25 \text { ans }\end{array}$ & $\begin{array}{l}\mathrm{de} 25 \\
35 \text { à } \\
35 \text { s }\end{array}$ & $\begin{array}{l}\text { de } \\
45 \text { ans }\end{array}$ & $\begin{array}{l}\text { de } 45 \\
55 \text { ans }\end{array}$ & $\begin{array}{c}\begin{array}{c}\text { plus } \\
\text { de }\end{array} \\
55 \text { ans }\end{array}$ & Total & $\begin{array}{c}\text { Total général } 2 \\
\text { des hommes } \\
\text { qualifiés et des } \\
\text { journaliers }\end{array}$ & $\begin{array}{l}\text { Pourcentage de } \\
\text { journaliers dans } \\
\text { la main-d'oeuvre } \\
\text { Total }\end{array}$ \\
\hline
\end{tabular}

(1) Ce total général porte seulement sur les 8 métiers principaux: charpentier, briqueteurs, plâtriers, plombiers, ferblantiers, chauffage, électriciens, peintres.

Il ressort clairement de ce tableau

a) que le nombre des journaliers est d'autant plus élevé que l'âge est plus bas, et que ce nombre décroit rapidement au fur et à mesure que l'âge s'élève.

b) Que, sauf dans le cas de Chicoutimi (où l'énorme superficie du territoire du comité paritaire explique la difficulté de l'apprentissage) on observe partout un pourcentage moyen oscillant entre 32 et $35 \%$ de journaliers dans la profession

c) Indiquons que dans tous les autres comités paritaires, dont les effectifs sont beaucoup plus faibles et dont nous ne publions pas les chiffres, les deux mêmes phénomènes s'observent.

Voici d'ailleurs la récapitulation pour toute la province:

\section{TABLEAU II}

Classification, par âge, des journaliers du bâtiment dans la province de Québec

\begin{tabular}{|c|c|c|c|c|c|c|c|c|}
\hline Territoire & $\begin{array}{c}\text { Moins } \\
\text { de } \\
25 \text { ans }\end{array}$ & $\begin{array}{l}\text { de } 25 \\
35 \text { à ans }\end{array}$ & $\begin{array}{l}\text { de } 35 \\
45_{\text {à }} \text { ans }\end{array}$ & $\begin{array}{c}\text { de } 45 \\
55 \text { ans }\end{array}$ & $\begin{array}{l}\text { plus } \\
\text { de } \\
55 \text { ans }\end{array}$ & Total & $\begin{array}{l}\text { Total général } \\
\text { de hommes } \\
\text { qualifiés et } \\
\text { des journaliers }\end{array}$ & $\begin{array}{c}\text { Pourcentage des } \\
\text { journaliers dans } \\
\text { la main-d'oeuvre } \\
\text { Total }\end{array}$ \\
\hline $\begin{array}{l}\text { Province } \\
\text { entière }\end{array}$ & 6,164 & 5,077 & 2,772 & 1,535 & 915 & 16,463 & 46,128 & $36 \%$ \\
\hline
\end{tabular}

Ce tableau confirme exactement les précisions fournies précédemment. Notons que le pourcentage général de $36 \%$ de journaliers dans l'ensemble de la main-d'oeuvre couverte par le recensement serait de $33 \%$, si le cas particulier de Chicoutimi ne venait rompre la constance que l'on observe dans les onze autres comités paritaires.

D'autre part, voici calculé sur $52 \%$ des ef- 
fectifs, la comparaison de l'âge moyen des hommes compétents et de celui des journaliers dans les principaux métiers:

\section{TABLEAU III}

Age moyen des hommes compétents par métier

\begin{tabular}{l|l}
\hline Plâtriers & 42 ans 9 mois \\
\hline Briqueteurs & 41 ans \\
\hline Charpentiers & 40 ans 4 mois \\
\hline Peintres & 39 ans \\
\hline Electriciens & 36 ans 10 mois \\
\hline Plombiers & 36 ans 4 mois \\
\hline Ferblantiers & 35 ans 3 mois \\
\hline
\end{tabular}

Et voici l'âge moyen des journaliers:

TABLEAU IV

\begin{tabular}{|l|c|}
\hline \multicolumn{2}{|c|}{ Age moyen des journaliers } \\
\hline Journaliers & 32 ans 6 mois \\
\hline
\end{tabular}

Il apparaît clairement, à première lecture, que tous les métiers ont un âge moyen qui oscille entre 35 ans et 43 ans, seul l'âge moyen des journaliers est inférieur à 33 ans. C'est dire que la proportion considérable de journaliers jeunes observée aux tableaux I et II n'est pas suivie par une proportion aussi élevé de compagnons jeunes.

\section{$\mathrm{B}$-Les causes}

L'étude faite des correlations entre les phénomènes précédents et les salaires, ainsi que l'enquête verbale établissent que, dans des proportions difficiles à déterminer, les phénomènes suivants sont à l'origine des faits observés:

a) la prime à l'incompétence résultant de la disparité entre le salaire du journalier qui débute à un taux plus élevé que l'apprenti -sans doute est-il légitime, du point de vue moral, que l'apprenti, qui acquiert un savoir, et donc rend moins de service et reçoit une compétence en même temps que de l'argent, gagne un salaire provisoirement inférieur Mais en fait, les jeunes, faute d'une formation suffisante, préfèrent un gain immédiat un peu plus élevé et négligent la rentabilité ultérieure du sacrifice provisoire de l'apprentissage; b) l'entrepreneur préfère utiliser le journalier plutôt que de former un apprenti, car l'apprenti lui coûte plus cher, non en salaire, mais en temps de formation. En outre, l'apprenti a tendance à changer de patron pour obtenir un salaire plus élevé dès qu'il commence à connaître le métier, et le patron n'a donc pas l'occasion de se voir récompenser par la fidélité économique de son apprenti de la compétence qu'il lui permet d'acquérir;

c) les contremaîtres et les compagnons ne sont généralement pas favorables à l'apprentissage, en particulier dans les métiers traditionnels. Il y a à cela deux causes principales. D'une part, le souvenir du chômage consécutif à la crise de 1928. C'est depuis cette époque que le quota dans ces métiers a été abaissé à $1 / 5$. D'autre part, la libre concurrence qui fait que les plus âgés qui sont aussi les plus compétents n'enseignent que quelques opérations faciles afin de «se garder le travail». Ils provoquent une prolétarisation des jeunes en vue de se prémunir eux-mêmes contre la crainte du chômage. Dans certains cas, on a vu le mélange des couleurs, dans la peinture, devenir un véritable secret que l'on ne livre pas aux apprentis, et qu'ignorent un grand nombre de compagnons.

A ces trois causes d'ordre économique où l'intérêt personnel immédiat des trois catégories: jeunes - entrepreneurs - compagnons, joue contre l'intérêt commun permanent de la profession et de la province correspondent trois causes d'ordre moral. En effet, les faits constatés montrent l'insuffisante formation:

a) des jeunes, qui généralement considèrent le gain immédiat et non le gain futur, ignorent l'importance psychologique et sociale d'avoir un métier bien en main, et enfin semblent incapables de l'acte de volonté de faire un sacrifice d'argent à un âge où, pour la plupart, ils n'ont pas encore de charge de famille;

b) d'un grand nombre d'entrepreneurs, qui sauf là où la création des Centres d'apprentissage comme celui de Montréal démontre le contraire - n'acceptent pas le sacrifice d'argent nécessaire à leur responsabilité morale de former des apprentis, qui correspond d'ailleurs à leur intérêt bien compris;

c) des compagnons qui s'imaginent à tort que l'accroissement du nombre des compagnons compétents fera baisser les salaires. (Ce sont les métiers mécaniques où le nombre des ap- 
prentis est le plus élevé, où les salaires sont eux-mêmes les plus élevés.)

\section{$\mathrm{C}$-Les remèdes}

Ils ont été clairement indiqués dans les voeux unanimes votés aux deux dernières réunions du Conseil de l'Apprentissage, ’̀ Sherbrooke et à Montréal. On peut les ramener à deux:

a) une campagne d'éducation auprès des jeunes, des contracteurs et des compagnons sur le plan moral;

b) le développement de la politique des centres dapprentissage et des écoles de préapprentissage sur le plan économique.

La loi de l'aide à l'apprentissage facilite la création de commissions d'apprentissage dont certaines ont créé des écoles ou centres de préapprentissage. Jusqu'ici de tels centres fonctionnent régulièrement à Montréal, Sherbrooke, Chicoutimi, et des cours du soir destinés à donner une formation théorique fonctionnent en outre à Hull, à Joliette et à Matane. Si l'on tient compte de ce qui a été dit plus haut au sujet des Trois-Rivières et de Rimouski, la seule ville importante, où existe d'ailleurs une commission d'apprentissage, mais où rien d'effectif ne semble en oeuvre en vue de la formation d'un centre, est Québec.

L'étude approfondie que nous avons faite, au cours de plusieurs visites dans les différents centres permet d'affirmer l'excellence de la formule qu'ils réalisent, et qui résultent de trois conditions remplies:

-les Centres d'apprentissage sont soutenus et dirigés non par l'Etat, mais par la profession sous le contrôle de l'Etat, d'où leur bonne adaptation en vue des fins à réaliser;

-l'équilibre de la formation théorique et de la formation pratique y est partout réalisé, compte tenu de différences secondaires résultant des conditions locales;

- la formation morale et sociale tient une place suffisante en vue de faire non seulement des apprentis compétents dans leur technique mais aussi consciencieux dans leur travail et enfin, ce qui n'est pas le moins important conscients de leur rôle social et économique.

Le préapprentissage ne résout pas, pour un apprenti, tout le problème de l'apprentissage, mais il lui donne une formation telle que:

a) l'entrepreneur ne craint plus de prendre un apprenti qui donnera un rendement très supérieur à un journalier; b) le compagnon a beaucoup plus de peine à cacher son savoir-faire à un apprenti déjà formé aux principales opérations du métier;

c) l'apprenti lui-même a conscience de sa supériorité technique et se trouve mieux à même de se perfectionner au cours de son apprentissage chez l'entrepreneur.

Ainsi, le Centre de préapprentissage résout partiellement certains des problèmes posés précédemment. Reste à déterminer l'importance quantitative de leur rôle, qualitativement si souhaitable.

Le tableau ci-dessous donne le total des apprentis sortis du Centre de préapprentissage de Montréal en regard du total des apprentis actuels dans chaque profession. Les autres centres de préapprentissage ne fonctionnent pas depuis un temps assez long pour que les statistiques soient révélatrices:

\section{TABLEAU V}

\begin{tabular}{l|c|c}
\multicolumn{1}{c|}{ Métiers } & $\begin{array}{c}\text { gradués ou } \\
\text { en cours } \\
\text { d'étude au } \\
30 \text { juin } \\
1948^{1}\end{array}$ & $\begin{array}{c}\text { Total des } \\
\text { apprentis } \\
\text { de 4 années } \\
\text { à Montréal }\end{array}$ \\
\hline $\begin{array}{l}\text { Charpente- } \\
\text { menuiserie }\end{array}$ & 141 & 313 \\
$\begin{array}{l}\text { Briquetage } \\
\text { Plâtrage }\end{array}$ & 204 & 128 \\
Ferblanterie & 69 & 129 \\
Plomberie et & 63 & 125 \\
Chauffage & 87 & 1,312 \\
Electricité & 96 & 789 \\
Peinture & 51 & 98 \\
\hline Total & 711 & 2,894 \\
\hline
\end{tabular}

Depuis l'ouverture en novembre 1945. Ces chiffres sont extraits du rapport de M. A.-E. Bourbeau, direteur du Centre, en date du 30 juin 1948

Comme on le voit, le Centre de préapprentissage de Montréal joue un véritable rôle de sauvetage dans la circonscription en ce qui concerne les métiers traditionnels. Le fait est remarquable en ce qui concerne les briqueteurs qui, si l'on tient compte des élèves inscrits au Centre, mais non encore en apprentissage, doivent la presque totalité de l'apprentissage au Centre. Il reste toutefois que l'effort de Montréal est encore insuffisant par rapport à la situation qui a été décrite au début de la seconde partie de ce travail, et que cet effort doit être encouragé par tous les moyens dans les autres Comités paritaires. 